九州大学学術情報リポジトリ

Kyushu University Institutional Repository

\title{
Miniaturization of Worm-type Soft Robot Actuated by Magnetic Field
}

\section{Maeda, Kazuki}

Department of Mechanical Engineering, Graduate School of Kyushu University

Shinoda, Hayato

Department of Mechanical Engineering, Graduate School of Kyushu University

Tsumori, Fujio

Department of Mechanical Engineering, Kyushu University

http://hdl. handle. net/2324/4403548

出版情報: Japanese Journal of Applied Physics. 59 (SI), pp.SIIL04-，2020-06-01. 応用物理学会 バージョン：

権利関係 : 


\title{
Miniaturization of Worm-type Soft Robot Actuated by Magnetic Field
}

\author{
Kazuki Maeda ${ }^{1}$, Hayato Shinoda ${ }^{1}$, and Fujio Tsumori ${ }^{2 *}$ \\ ${ }^{1}$ Dept. Mech. Eng., Grad. School of Kyushu Univ., 744 Motooka, Nishi-ku, Fukuoka \\ 819-0395, Japan \\ ${ }^{2}$ Dept. Mech. Eng., Kyushu Univ., 744 Motooka, Nishi-ku, Fukuoka 819-0395, Japan \\ E-mail: *tsumori@mech.kyushu-u.ac.jp
}

Recently, many studies on bio-mimic soft robots have been reported. Among living organisms, locomotion of soft bodies, such as snails, worms, and nematodes, have been actively studied. These soft creatures locomote by deforming the muscles of the whole body into waveforms and propagating the waves of the deformation. These locomotion of soft creatures could be applicable to develop a worm-type soft robot which can move in drain pipes and even if in a human body. In this study, we developed worm-like robots which could generate wavy motion under a rotating magnetic field using silicone and magnetic rubber, and investigated its movement characteristics. In addition, based on deformation experiment of a silicone cantilever with a single magnetic element inside made of the same material of worm-like robot, we investigated the effect of the size of the worm robot for further miniaturization. 


\section{Introduction}

In recent years, soft robotics has been focused as a new field to realize various soft and flexible motions ${ }^{1-11)}$. In this soft robotics field, robotic bodies without rigid frames have been developed so that they would not hurt anything if they touch it. Also, the soft bodies are attractive especially to mimic soft motion of natural organisms. The natural flexible motions could be useful since living creatures have excellent functions obtained by years of their evolution. Among living organisms, locomotion of soft bodies, such as snails, worms, and nematodes, have been actively studied ${ }^{12)}$. In this work, we focused on these locomotion of soft creatures, which could be applicable to develop a worm-type soft robot which can move in drain pipes and even if in a human body.

These soft creatures locomote by deforming the muscles of the whole body into waveforms and propagating the waves of the deformation. Niebur ${ }^{13}$ ) has performed simulation of wavy motion to express locomotion of soft bodies. Sauvage ${ }^{14)}$ and Tanaka ${ }^{15)}$ have showed that phase shift between propagations of muscle wave and friction wave was important factor for the locomotion. Ijspeert ${ }^{16)}$ and $\mathrm{Crespi}^{17)}$ have developed amphibious locomotion robots using motors. Yuk ${ }^{18)}$ have developed a robot which locomoted with a thermal deformation spring.

In this study, we aimed to develop a miniaturized soft robot that can be actuated in small gaps or in soft bodies. For this purpose, we employed silicone elastomer and rubber magnetic material to downsize the robot. Magnetic elastomer could be used for many purposes, for example, dynamic control of the mechanical properties ${ }^{19)}$, energy harvesting ${ }^{20)}$, and of course various kinds of soft actuators ${ }^{21-40)}$. There have been many reports for bio-mimic approach using magnetic elastomers, such as artificial cilia ${ }^{27-34)}$ and worm-like $\operatorname{robots}^{33,34)}$. There are advantages for these magnetic soft actuators; the energy can be transmit without electric wiring so that the structure could be simple and easy to be downsized. In this work, we will discuss about miniaturization of soft robots developing worm-like robots which could generate wavy motion under a rotating magnetic field.

\section{Materials and Methods}

We performed 2 experiments in this work. The first one was an experiment using a simple rubber cantilever structure with 1 magnetic element. The other one was actuation of 
worm-type robots between gaps. Details of these experiments described in the following sections.

\section{Bending of Single Cantilever}

We conducted a simple deformation experiment of a silicone cantileve with a single magnet element inside to know the simplest deformation of the structure. In this experiment, we applied an external magnetic field to the beam, and measured the $\mathrm{x}$ displacement. We used platinum-catalyzed silicone elastomer (Ecoflex 0010, Smooth-On) as base material. Also, we prepared a commercial rubber magnet (Daiso), and magnetized the magnet using vibrating sample magnetometer (VSM) at $1.56 \mathrm{~T}$. A schematic image of the experiment is shown in Fig. 1. The cantilever was set between 2 permanent magnets to apply a magnetic field. The permanent magnets were rotated to change the direction of the magnetic field. We designed 2 sizes of beams, $30.0 \mathrm{~mm} \times 10.0 \mathrm{~mm} \times 3.0 \mathrm{~mm}$ and $15.0 \mathrm{~mm} \times 5.0 \mathrm{~mm} \times 1.5$ $\mathrm{mm}$. Rubber magnets for the beams were cut to $1.0 \mathrm{~mm} \times 1.0 \mathrm{~mm} \times 10.0 \mathrm{~mm}$ and $0.5 \mathrm{~mm} \times$ $0.5 \mathrm{~mm} \times 5.0 \mathrm{~mm}$, respectively. The beams were made using acrylic molds, and the magnets were fixed near the tops of the beams. The Young's modulus of the silicone elastomer was less than $50 \mathrm{kPa}$, and the residual magnetic flux density of the magnetic rubber was 1.22 $\mathrm{Wb} / \mathrm{m}^{2}$. We applied the external magnetic field to the beam at 15 degrees from 0 to 360 degrees and measured the displacement at that time. The external magnetic field strength was 20,40 , and $60 \mathrm{mT}$.

\section{Worm-type robot}

We designed worm-type robot as shown in Fig. 2. The worm deformed into a wavy shape and the wave would propagate as rotating the magnetic field. Flow of the fabricating method is shown in Fig. 3. We used the same materials explained in the previous experiment. The rubber magnet blocks were arranged in a plastic frame changing each magnetic direction by 90 degrees. Then, the silicone was poured around the magnetic material blocks and cured by heat. In this study, we fabricated 2 different sizes of worm-type robots; one was $37 \mathrm{~mm}$ long $\times 8.5 \mathrm{~mm}$ wide $\times 1.5 \mathrm{~mm}$ thick, and the other was $19 \mathrm{~mm}$ long $\times 4.3 \mathrm{~mm}$ wide $\times 0.75$ $\mathrm{mm}$ thick. The magnetic elements placed inside the robot was $1.0 \mathrm{~mm} \times 1.0 \mathrm{~mm}$ and $5 \mathrm{~mm}$ wide prism blocks for the larger robot, and $0.5 \mathrm{~mm} \times 0.5 \mathrm{~mm}$ and $2.5 \mathrm{~mm}$ wide prism blocks for the smaller one. Eight prism blocks were used for each robot. 


\section{Results and Discussions}

\section{Bending of Single Cantilever}

Snapshots of the bent beam are shown in Fig. 4. The cantilever bent to left direction as the applied magnetic field rotated to anticlockwise, and it snapped to right direction around at $180^{\circ}$. Theoretical angle $\theta$ of the tip of the cantilever can be obtained from the following equation:

$$
E I \theta / L=m B \sin \left(\theta-\theta_{\mathrm{B}}\right),
$$

where, $E$ is Young's modulus, $I$ is cross-section second moment of the beam, $L$ is the beam legnth, $m$ is residual magnetic flux density of the magnetic element, and $B$ and $\theta_{\mathrm{B}}$ are the intensity and the angle of an applied magnetic field, respectively. Only the dimensionless value of $E I / m B L$ determins this equation. Once $\theta$ is obtained, also the displacement of the tip could be calculated. Figure 5 shows the $x$-displacement of the tips of the smaller and larger cantilevers under a magnetic field of $20 \mathrm{mT}$. The displacement was normalized by the length of the beam. The theoretical curve is also plotted in this figure. We selected the value of $E I / m B L$ as 2.70 to fit the experimental result.

As expected from the theoretical formulation, the smaller and the larger samples deformed similarly. The difference found at the peak and the valley points would be caused by an inhomogeneous magnetic field. It would be needed to prepare larger setup to apply a homogeneous magnetic field to the larger sample. Figures 6 and 7 show $x$-displacements of the smaller sample under 40 and $60 \mathrm{mT}$. Theoretical curves are also shown here. The used values are 1.35 and 0.90 for 40 and $60 \mathrm{mT}$, respectively, which are half and one third of 2.70 used for the condition of $20 \mathrm{mT}$. The experimental results showed good agreements with theoretical values. We can verify the theoretical equation from these results. Equation (1) shows that similar deformation would be obtained even if the size of the samples were different. It means that further miniaturization of the present system could be obtained.

\section{Worm-type robot}


Next, we demonstrate worm-type robots. Fabricated robot sample was put between the gaps as shown in Fig. 8. Figure 9 shows snapshots of the actuated smaller and larger samples beween gaps of 2 and $4 \mathrm{~mm}$, respectively. The external magnetic field strength was $85 \mathrm{mT}$, and paper (KimWipes, Nippon-paper Cresia) was set on the wall surface to prevent adhesion between the robot and the wall glass material. The samples deformed similarly into a wave shape as designed, and the waves propagated as the rotation of the external magnetic field. This similarity was expected from the previous formulation.

Figure 10 shows the relationship between the rotational speed of the external magnetic field and the locomotion speed normalized by each body length of the robot. Almost linear relationship was obtained for each sample. There was some deviated part in the case of the smaller sample around 100 to $150 \mathrm{rpm}$. It might be near the natural frequency of the smaller sample. Figure 11 shows the change of the locomotion speed as the magnetic field strength was changed from 0 to $100 \mathrm{mT}$. Both smaller and larger samples increased in speed as the magnetic field strength increased. The smaller and larger samples moved almost without touching the upper wall from 0 to $40 \mathrm{mT}$. After then, the compressive force to the walls increased as the magnetic field increased. In this case, the effect of the friction, which prevented the locomotion, on the smaller sample would be larger. Figure 12 shows the locomotion speed as the gap was changed. The gap was normalized by the thickness of each sample body. Also, in this case, the larger sample was faster than the smaller one.

We explained in the previous section that the deformation of the cantilevers would be similar even if the size was changed. Also, almost similar results of the actuation tests were obtained for the smaller robot and the larger one. This means that the motion is independent on the size in this system.

The final data was taken changing the wall surface state. We prepared 3 kinds of wall surfaces, KimWipes, which was the same condition as used in the above experiments, printing paper used for office works, and glass substrate. The SEM images of the papers are shown in Fig. 13. Rougher and finer fiber structures are observed. These surface states could change the frictional or adhesive interaction. We checked the difference of the interaction using a setup as shown in Fig. 14. Two kinds of surfaces were prepared and a PDMS sheet was set to bridge the both surfaces. We could know which force was larger by pulling the 
substrate sideways. As the result, the adhesive or frictional force of the glass surface was largest, the printing paper was 2 nd, followed by KimWipes.

Also, 3 sizes of robots were prepared. The length of the larger and smaller ones were 37 and $19 \mathrm{~mm}$, which were same with the previous experiments. The size between them was also prepared, which was $26 \mathrm{~mm}$ in length. Three robots were actuated between walls of 3 kinds of the prepared surfaces. Some samples locomoted, but others were sticked on the surface. Table 1 summarizes the results of the actuation. Some difference would be caused by the adhesion and the friction. The larger the adhesion, the more difficult the locomotion. So, any sample could not locomote on the glass surface, while the all samples could on the KimWipes. On the printing paper, which had middle adhesion state, only the largest robot could work. As the structure miniaturized, the effect of the surface, such as adhesion and friction, could be larger. We should consider this effect for further miniaturization of the robot.

To downsize the robot, we also need a better fabrication tool. We are now working on development of a 3D printing system for magnetic soft robotics ${ }^{34-37)}$. UV curable elastomer or gel material is employed for this system. This flexible material is mixed with magnetic particles and used for the similar process to conventional 3D printing systems. During the 3D-building process, a magnetic field is controlled to set anisotropic orientation of magnetization.

\section{Conclusions}

In this study, we fabricated magnetic driven worm-type robots. We also performed some simple actuation tests of cantilevers using the same material to show it would be possible to miniaturize the system. The larger and smaller structures deformed similarly as expected the proposed theoretical model. The results of the larger and smaller locomotion robots showed also similarity except on the effect of the adhesion and the friction.

For further miniaturization, we should consider the effect of the surface effect, and also to develop a new fabrication tools. We are now developing 3D printing system for the magnetic elastomer or gel material ${ }^{34-37)}$, which would be a powerful tool for this purpose. Also, it would be essential to establish numerical approach to design the motion of magnetic 
soft actuators ${ }^{38-40)}$.

\section{References}

1) Y. Yang, X. Song, X. Li, Z. Chen, C. Zhou, Q. Zhou, and Y. Chen, Adv. Mater. 30, 1706539 (2018).

2) S. Kim, C. Laschi, and B. Trimmer, Trends Biotechnol. 31, 287 (2013).

3) R. Pfeifer, M. Lungarella, and F. Iida, Commun. ACM. 55, 76 (2012).

4) L. Ricotti, B. Trimmer, A. Feinberg, R. Raman, K. Parker, R. Bashir, M. Sitti, S. Martel, P. Dario, and A. Menciassi, Sci. Robot. 2, 1 (2017).

5) D. Rus, and M. Tolley, Nature 521, 467 (2015).

6) C. Laschi, B. Mazzolai, and M. Cianchetti, Sci. Robot. 1, 1 (2016).

7) L. Hines, K. Petersen, G. Lum, and M. Sitti, Adv. Mater. 29, 1603483(2017).

8) S. Rich, R. Wood, and C. Majidi, Nat. Electron. 1, 102 (2018).

9) A. S. Gladman, E. A. Matsumoto, R. G. Nuzzo, L. Mahadevan, and J. Lewis, Nat. Mater. 15,413 (2016).

10) F. Momeni, S. M. Mehdi, X. Liu, and J. Ni, Mater. Design. 122, 42 (2017).

11) B. Gorissen, D. Reynaerts, S. Konishi, K. Yoshida, J. W. Kim, and M. De Volder, Adv. Mater. 29, 1604977 (2017)

12) J. Aguilar, T. Zhang, F. Qian, M. Kingsbury, B. McInroe, N. Mazouchova, C. Li, R. Maladen, C. Gong, M. Travers, R. Hatton, H. Choset, P. Umbanhowar, and D. Goldman, R. Prog. Physics. 79, 1 (2016).

13) E. Niebur, and P. Erdos, Biophys. J. 60, 1132 (1991).

14) P. Sauvage, M. Argentina, J. Drappier, T. Senden, J. Sime'on, and J. Meglio, J. Biomech. 44, 1117 (2011).

15) Y. Tanaka, K. Ito, T. Nakagaki, and R. Kobayashi, J. Roy. Soc. Interface. 9, 222 (2011).

16) A. Ijspeert, A. Crespi, and J. Cabelguen, Neuroinformatics. 3, 171 (2005).

17) A. Crespi, and A. Ijspeert, IEEE Tran. Robot. 24, 1 (2008).

18) H. Yuk, D. Kim, H. Lee, S. Jo, and J. H. Shin, Bioinspir. Biomim. 6, 046002 (2011).

19) D. Maede, F. Tsumori, T. Osada, and K. Kudo, IEEJ Trans. Sensors Micromachines. 138, 48 (2018) [in Japanese].

20) H. Shinoda, and F. Tsumori, Jpn. J. Appl. Phys. 57, 06HJ05 (2018). 
21) J. Kim, S. E. Chung, S. E. Choi, H. Lee, J. Kim, and S. Kwon, Nat. Mater. 10, 747 (2011).

22) J. F. Zhou, C. L. Shao, and B. Q. Gu, J. Nanopart. Res. 18, 29 (2016).

23) J. Ku, H. Chen, K. He, and Q. Yan, Miner. Eng. 79, 10 (2015).

24) Hu, G. Z. Lum, M. Mastrangeli, and M. Sitti, Nature 554, 81 (2018).

25) F. Tsumori, and J. Brunne, Proc. IEEE MEMS 2011, 1245.

26) K. Hatama, F. Tsumori, Yang Xu, Hyungoo Kang, Toshiko Osada, and Hideshi Miura, Jpn. J. Appl. Phys. 51, 06FL14 (2012).

27) F. Tsumori, A. Saijou, T. Osada, and H. Miura, Jpn. J. Appl. Phys. 54, 06FP12 (2015).

28) F. Tsumori, R. Marume, A. Saijou, K. Kudo, T. Osada, and H. Miura, Jpn. J. Appl. Phys. $55,06 \mathrm{GP} 19(2016)$.

29) R. Marume, F. Tsumori, K. Kudo, T. Osada, and K. Shinagawa, Jpn. J. Appl. Phys. 56, 06GN15 (2017)

30) S. Azukizawa, F. Tsumori, H. Shinoda, K. Tokumaru, K. Kudo, and K. Shinagawa, Proc. MicroTAS (2017), 623.

31) H. Shinoda, S. Azukizawa, and F. Tsumori, Proc. MicroTAS (2018) 679.

32) H. Shinoda, and F. Tsumori, Proc. MEMS (2020) 497.

33) M. Furusawa, K. Maeda, S. Azukizawa, H. Shinoda, and F. Tsumori, J. Photopolym. Sci. Tech. 32, 309 (2019).

34) H. Shinoda, S. Azukizawa, K. Maeda, and F. Tsumori, J. Electrochem. Soc. 166, B3235 (2019).

35) F. Tsumori, H. Kawanishi, K. Kudo, T. Osada, and H. Miura, Jpn. J. Appl. Phys. 55, 06GP18 (2016).

36) S. Azukizawa, H. Shinoda, K. Tokumaru, and F. Tsumori, J. Photopolym. Sci. Tech. 31, 139 (2018).

37) S. Azukizawa, H. Shinoda, and F. Tsumori, Proc. IEEE-MEMS 2019, 248.

38) F. Tsumori, N. Miyano, and H. Kotera, Funtai Oyobi Funmatsu Yakin, 56, 133 (2009) [in Japanese].

39) F. Tsumori, N. Miyano, and H. Kotera, Funtai Oyobi Funmatsu Yakin, 56, 127 (2009) [in Japanese].

40) F. Tsumori, K. Hatama, H. Kang, T. Osada, and H. Miura, Proc. IEEE-NEMS 2013, 845. 


\section{Figure and Table Captions}

Fig. 1 (Color online) Schematic image of the simple cantilever deformation test. As the rotation of two permanent magnets, the cantilever deforms.

Fig. 2 (Color online) Schematic diagram of wavy motion in a worm-type soft robot.

Fig. 3 (Color online) Flow of fabricating method.

Fig. 4 (Color online) Snapshots of deformed beam with changing angle of an applied magnetic field from 0 to 360 degree.

Fig. 5 (Color online) X-displacement of tips of smaller and larger beams under an applied magnetic field of $20 \mathrm{mT}$.

Fig. 6 (Color online) X-displacement of smaller beam's tip under an applied magnetic field of $40 \mathrm{mT}$

Fig. 7 (Color online) X-displacement of smaller beam's tip under an applied magnetic field of $60 \mathrm{mT}$

Fig. 8 (Color online) Schematic image of experimental setup for actuation of the worm-type robots. Rotational magnetic field was applied by a permanent magnet.

Fig. 9 (Color online) Snapshots of worm-type robots under a rotational magnetic field of 30 rpm. Intensity of the magnetic field was $85 \mathrm{mT}$.

Fig. 10 (Color online) Relationship between normalized velocity of robot and rotational magnet speed.

Fig. 11 (Color online) Relationship between normalized velocity of robot and intensity of applied magnetic field. 
Fig. 12 (Color online) Normalized distance per cycle as changing wall gaps.

Fig. 13 SEM images of KimWipes (a) and printing paper (b), which were used for substrates.

Fig. 14 (Color online) Schematic of experimental setup for comparison of adhesive forces between PDMS sheet and substrates.

Table 1 Locomotion states of 3 crawler samples on walls of glass, printing paper, and KimWieps substrates. 


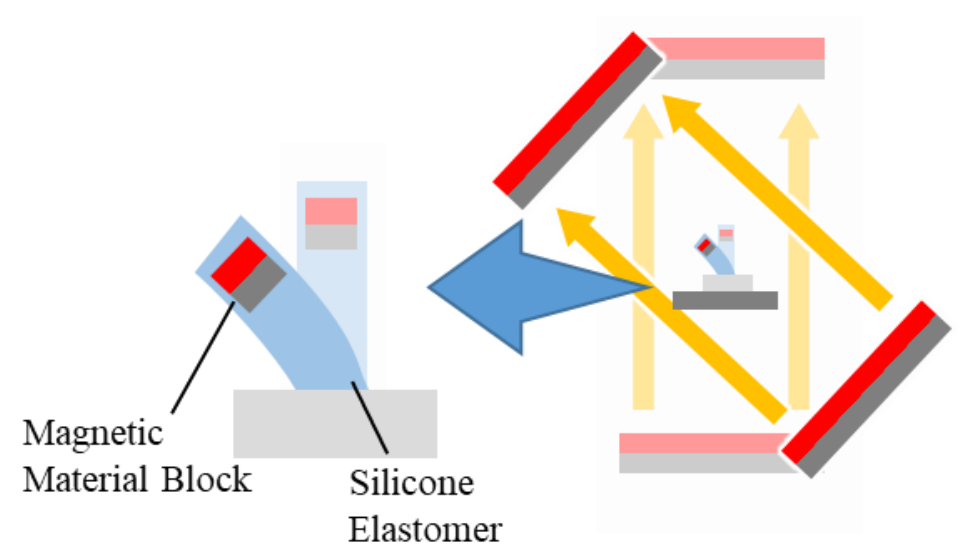

Fig. 1 (Color online) 


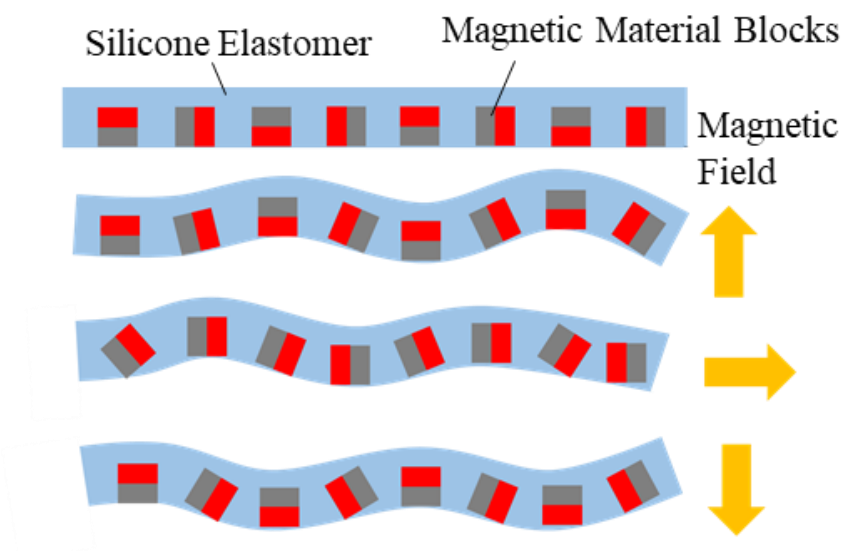

Fig. 2 (Color online) 
Magnetic

(1) Set up Magnetic Material Blocks Material Block

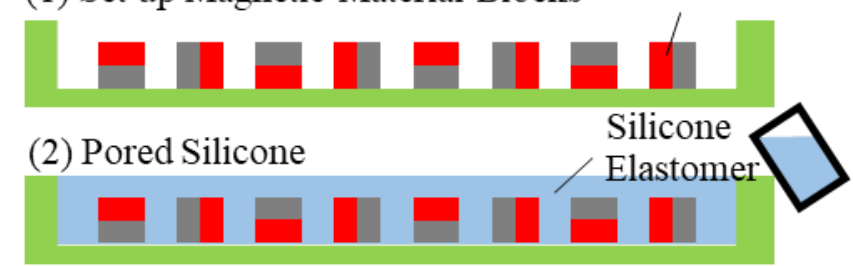

(3) Cured Silicone by Heat

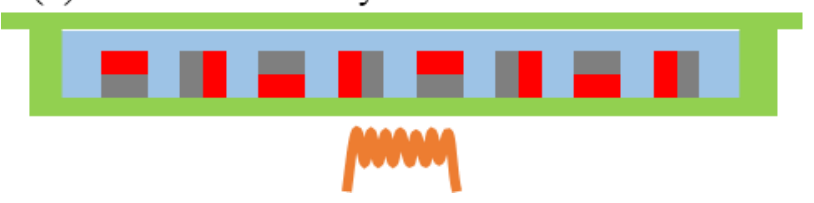

Fig. 3 (Color online) 

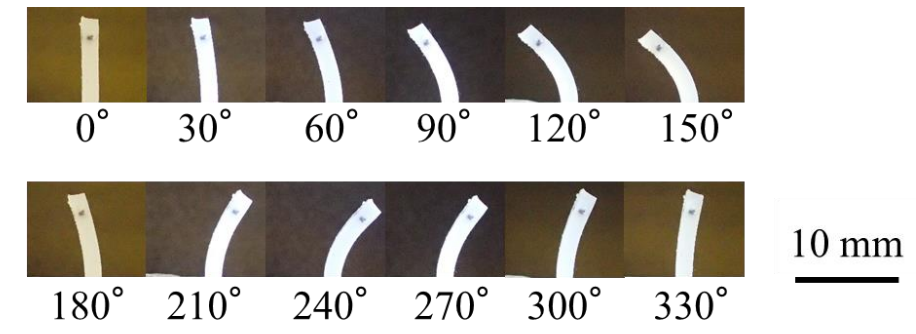

Fig. 4 (Color online) 


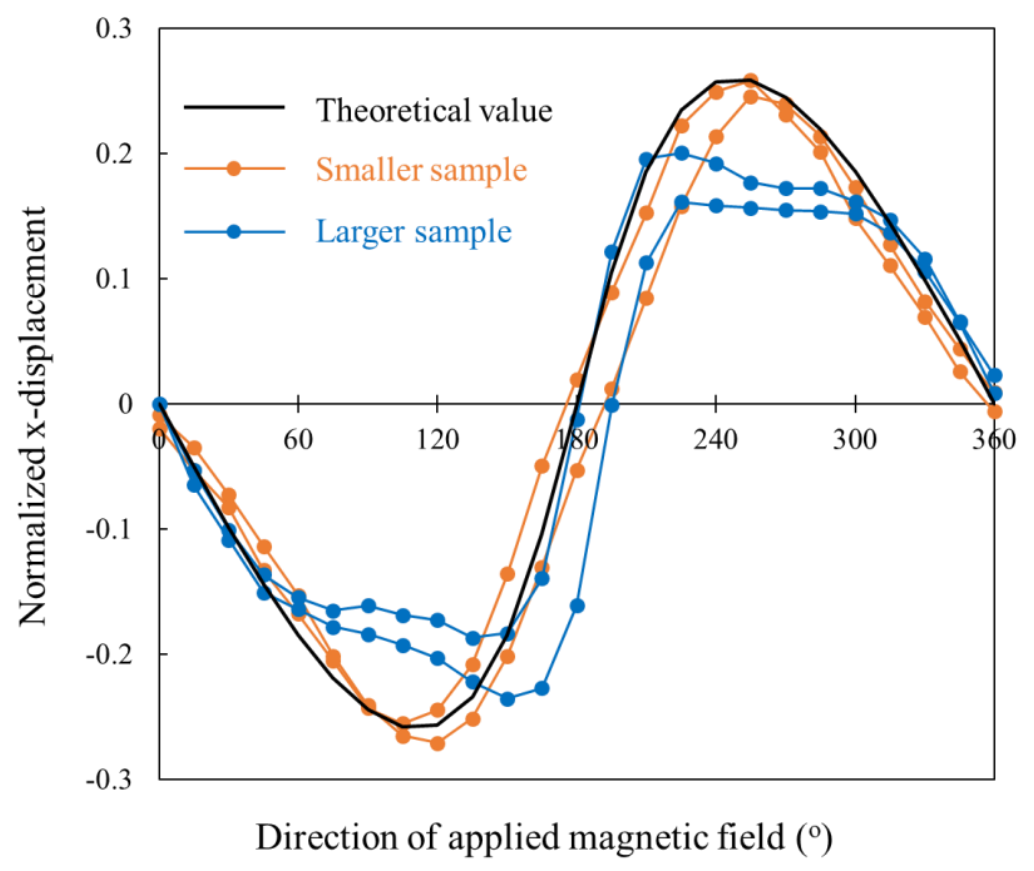

Fig. 5 (Color online) 


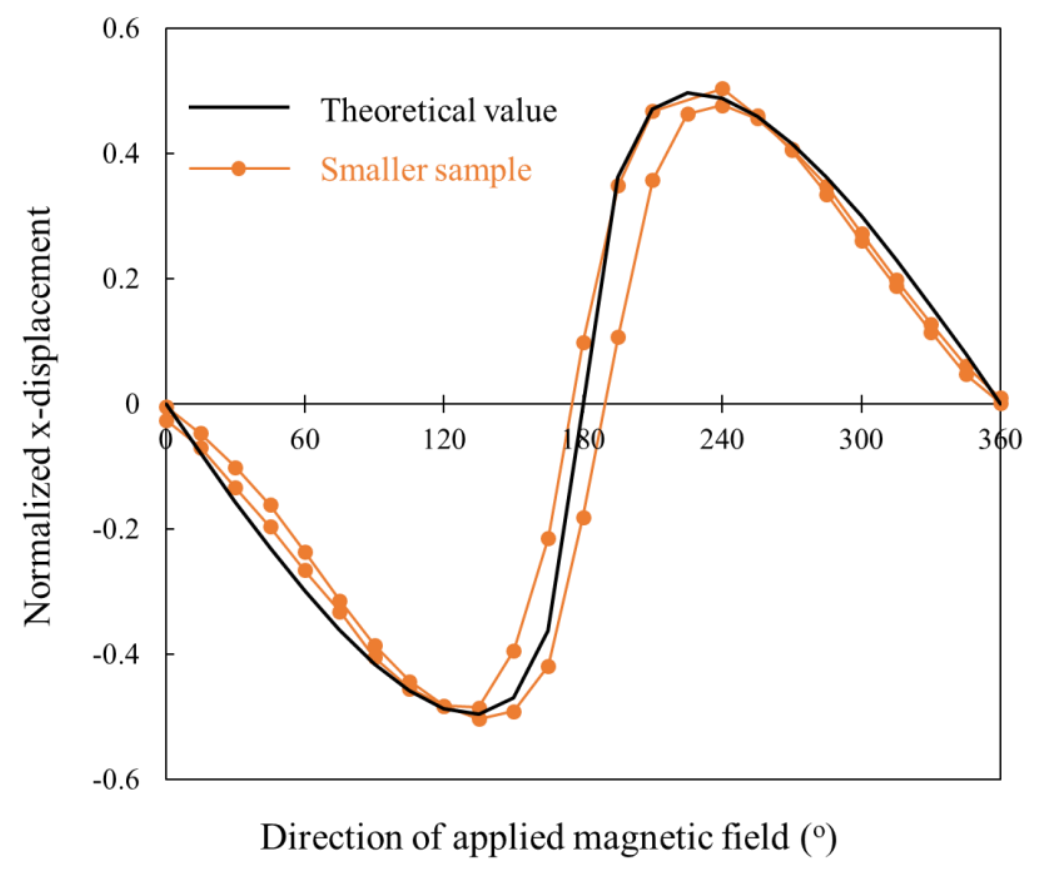

Fig. 6 (Color online) 


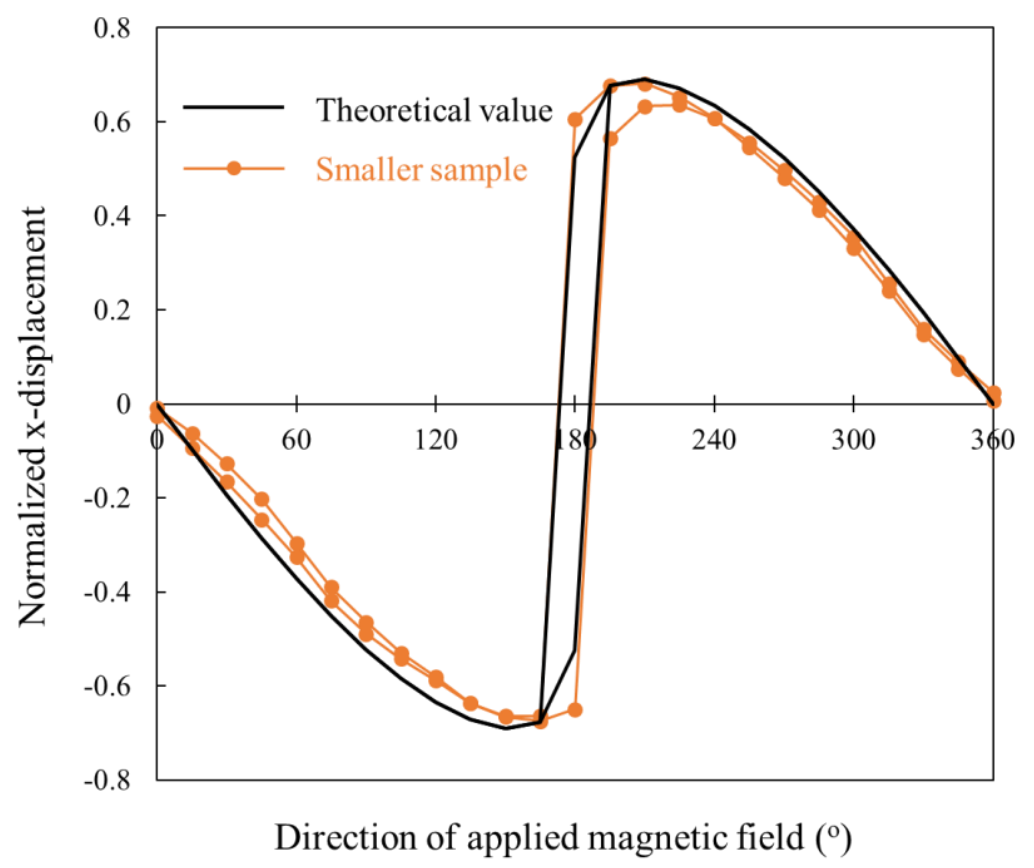

Fig. 7 (Color online) 


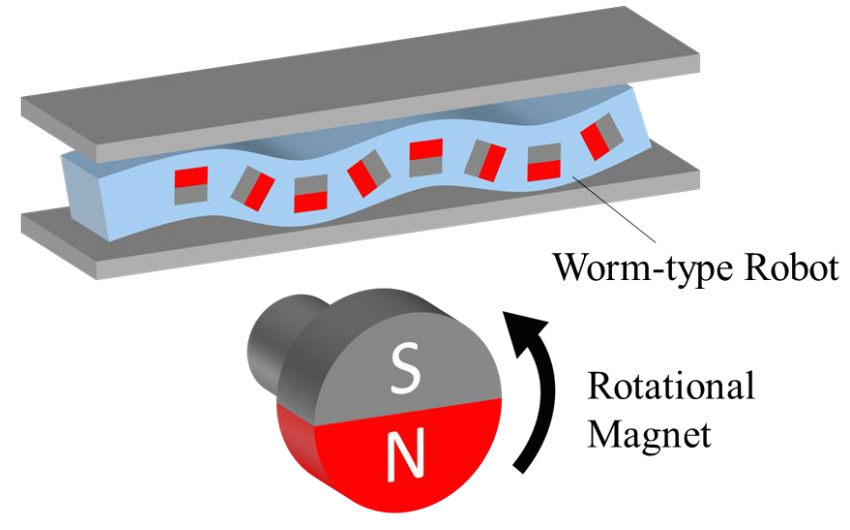

Fig. 8 (Color online) 

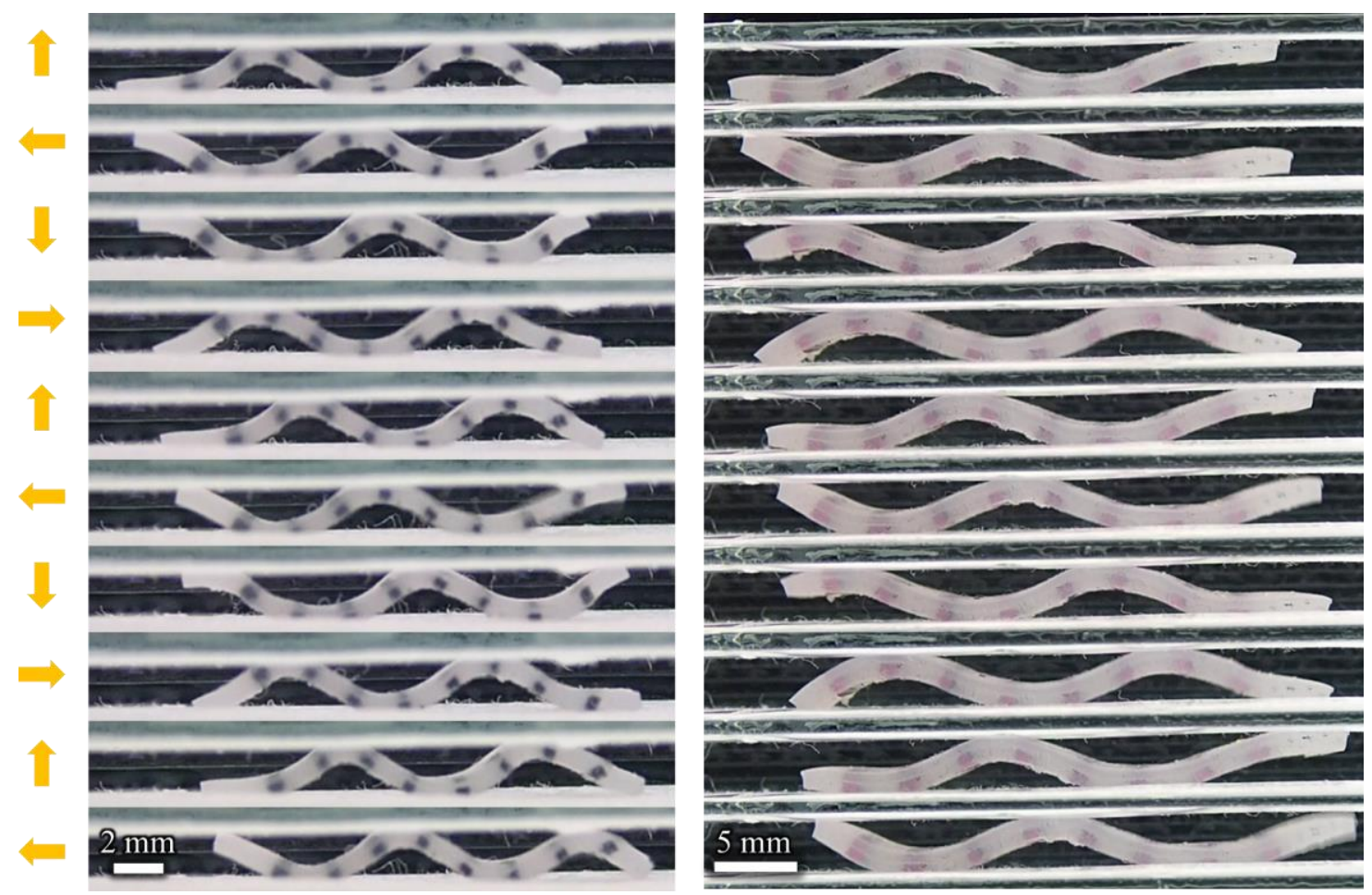

Fig. 9 (Color online) 


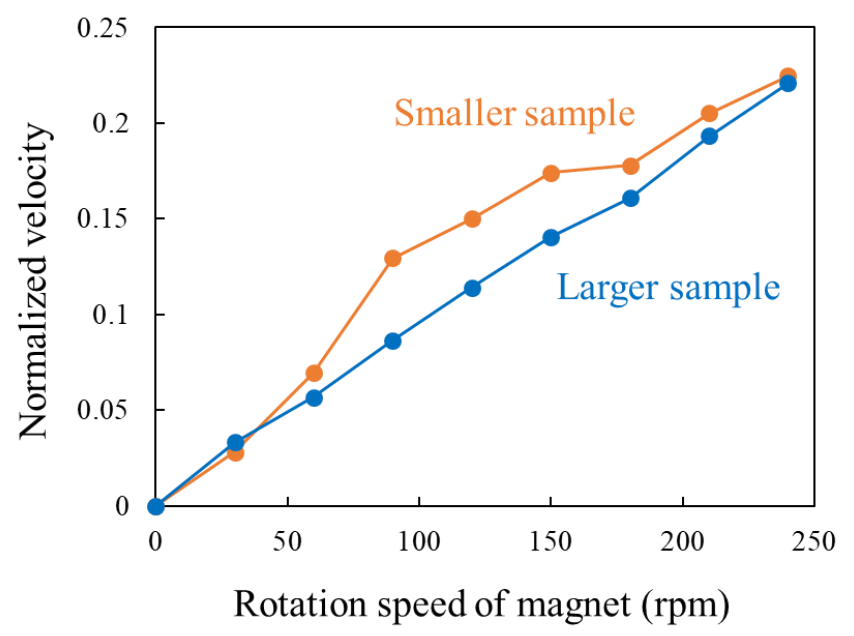

Fig. 10 (Color online) 


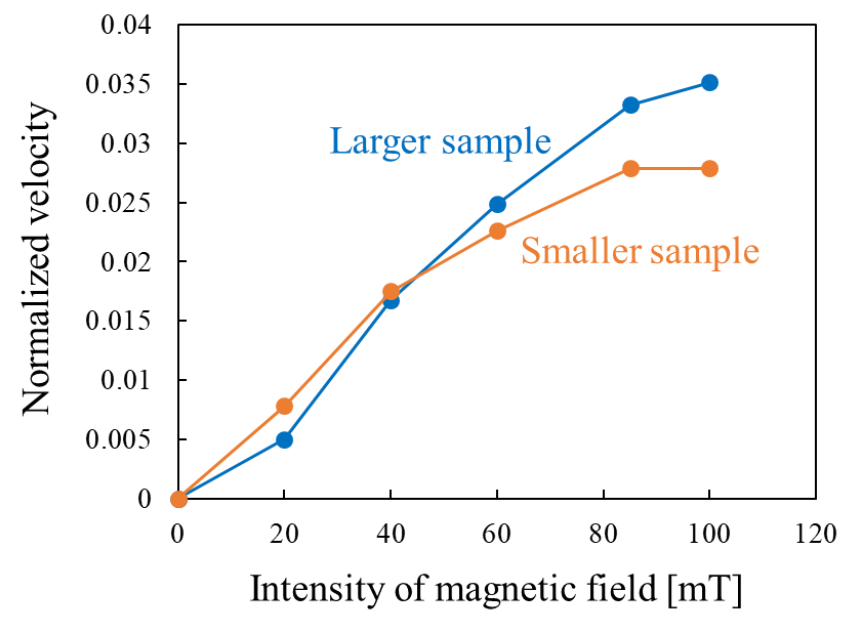

Fig. 11 (Color online) 


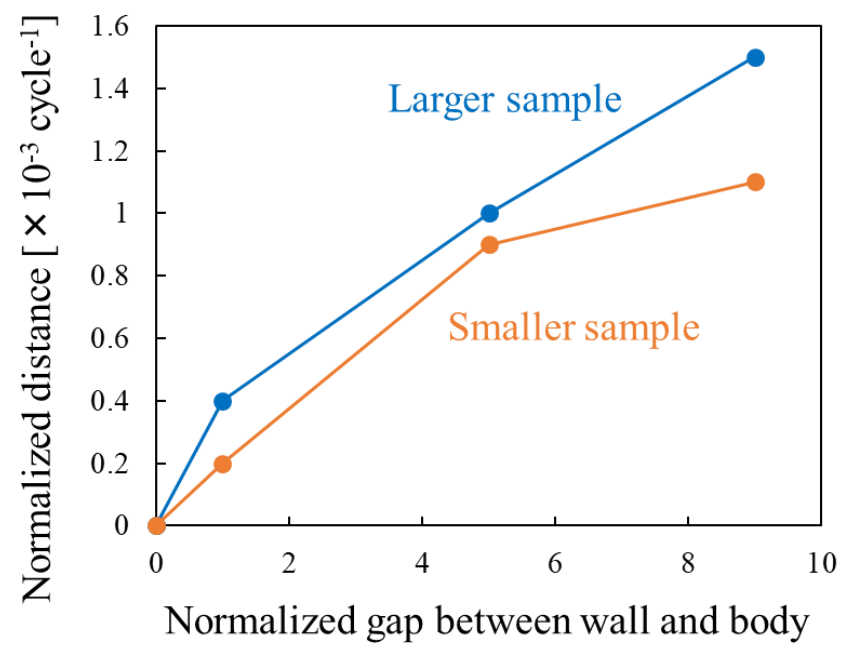

Fig. 12 (Color online) 

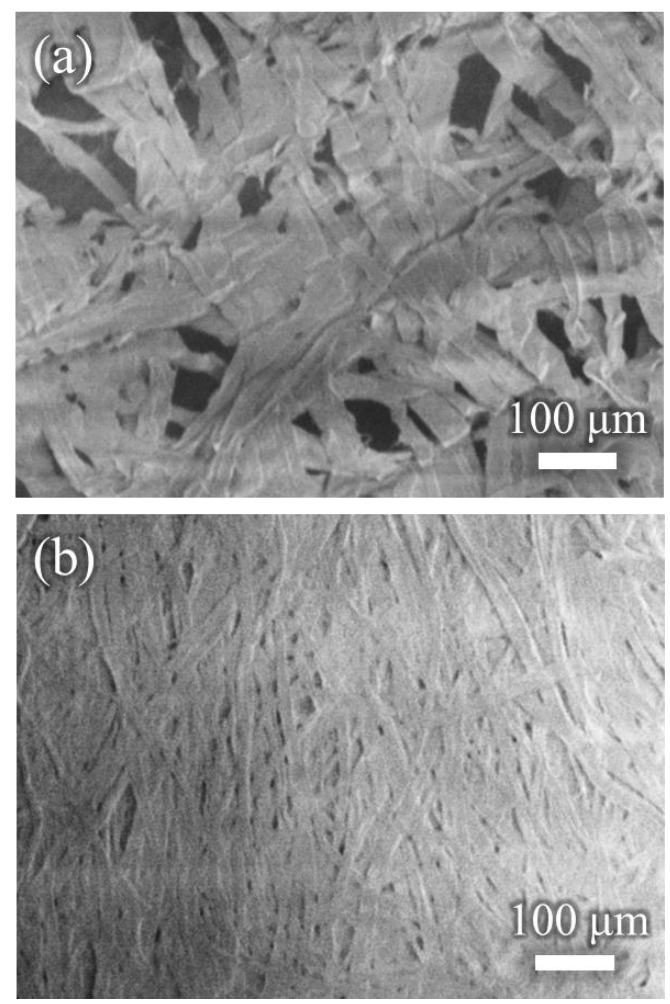

Fig. 13 


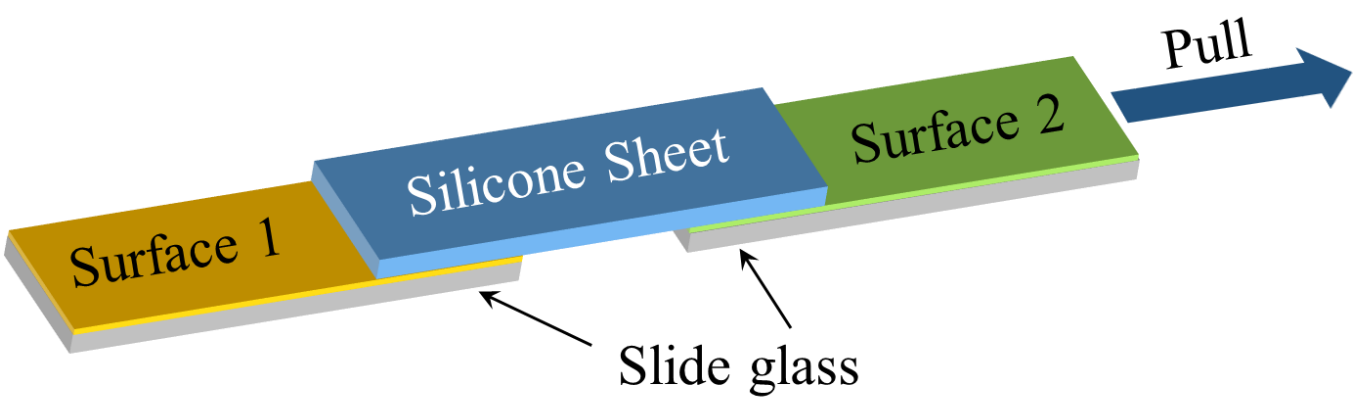

Fig. 14 (Color online) 
Table 1

\begin{tabular}{lccc} 
& Smaller & Middle & Larger \\
\hline Glass & $\times$ & $\times$ & $\times$ \\
Printing paper & $\times$ & $\times$ & 0 \\
KimWipes & 0 & 0 & 0 \\
\hline
\end{tabular}

